\title{
EPISOUTH: A NETWORK FOR COMMUNICABLE DISEASE CONTROL IN THE MEDIterRanean REgION AND THE BALKANS
}

\author{
M G Dente (mariagrazia.dente@iss.it) ${ }^{1}$, M Fabiani ${ }^{1}$, R Gnesotto ${ }^{2}$, G Putoto², C Montagna ${ }^{2}$, F Simon-Soria ${ }^{3}$, C Martin de Pando ${ }^{3}$, \\ P Barboza 4 , F Ait-Belghiti ${ }^{4}$, M Kojouharova5, N Vladimirova5, R Vorou ${ }^{6}, K_{\text {Mellou }}^{6}, \mathrm{G}$ Thinus 7 , S Declich1, for the EpiSouth \\ Network ${ }^{8}$
}

1. Istituto Superiore di Sanità (National Institute of Health), National Centre for Epidemiology, Surveillance and Health Promotion, Rome, Italy

2. Padua Teaching Hospital, Training and International Projects Department, Padua, Italy

3. Instituto de Salud Carlos III, (Carlos III Health Institute), National Epidemiology Centre, Madrid, Spain

4. Institut de Veille Sanitaire (French Institute for Public Health Surveillance), Department of International and Tropical

Diseases, Saint-Maurice, France Centre, Madrid, Spain

5. National Center of Infectious and Parasitic Diseases, Department of Epidemiology and Surveillance of Communicable Diseases, Sofia, Bulgaria

6. Hellenic Center for Disease Control and Prevention, Office for Zoonoses and Foodborne Diseases, Athens, Greece

7. European Commission, Directorate-General for Health and Consumer Protection (DG-Sanco), Luxembourg, Luxembourg

8. The Members of the EpiSouth Network are listed at the end of the article

The countries around the Mediterranean Sea share epidemiological characteristics and public health problems. In 2006 the EpiSouth Project was started as a framework for collaboration for communicable diseases surveillance and training in the Mediterranean Basin. As of December 2008, 26 countries from southern Europe, the Balkans, North Africa and the Middle-East are members of EpiSouth and several international organisations and institutions collaborate: the European Commission (EC), the European Centre for Disease Prevention and Control (ECDC), the Italian Ministry of Work, Health and Social Policies and the World Health Organization (WHO). The project is coordinated by the Italian national public health institute and three work packages (WPs) Cross-border epidemic intelligence, vaccine preventable diseases and migrants and Cross-border emerging zoonoses are operated by the national institutes of France, Bulgaria and Greece. These WPs constitute technical pillars on which the project develops. Networking and Training are WPs dedicated to capacity building and are run by the Padua Teaching Hospital (Italy) and the Spanish national public health institute. A steering committee guides EpiSouth's activities while all countries collaborate through WP steering teams and focal points. A number of outcomes have been accomplished and documents with results are available from the EpiSouth website which hosts a public website and a restricted area for direct sharing of information among the participants. Five electronic bulletins were published, two trainings for 63 participants performed, national epidemic intelligence systems were evaluated, a preliminary survey on vaccine-preventable diseases and migrants performed, and a list of priorities for emerging zoonoses in the Mediterranean area was selected. Overall the network succeeded in creating cohesion, mutual trust and concrete collaboration on cross-border public health issues in a geographical area that is not addressed as a whole by any other initiative or organisation.

\section{Background}

Infectious diseases are threats to human health that can rapidly spread across geographical regions and borders. Today, a number of them are preventable through effective and safe vaccines however, the majority are still prevented and controlled through a comprehensive approach, in which surveillance plays a crucial role. In order to initiate appropriate public heath action, early detection of cases, dissemination of information, and cross-border, harmonic and prompt response are crucial. The countries of the Mediterranean region have common sea borders in the ecosystem of the Mediterranean Sea and share public health problems. To address these common problems, in 2004, experts from Bulgaria, Greece, France and Italy designed a co-operative initiative covering the Mediterranean region and the Balkans, similar to that developed by the EpiNorth Project for the Baltic Region [1]. Spain soon joined the initiative and on occasion of the Year of the Mediterranean, in 2005, the project called EpiSouth was proposed to the countries in this area and to the European Commission (EC) for funding. EpiSouth was officially launched on 1 October 2006 and receives funding until 30 September 2009. The general objective of the project is to create a framework for collaboration on epidemiological issues to improve communicable diseases surveillance and communication and provide training for public health experts in the participating countries.

\section{Methods}

To enhance communicable disease control capacity in the Mediterranean region and the Balkans priority areas for activities were identified. Work in these areas is conducted through specific work packages (WP) and EpiSouth is focussing on the WPs Crossborder epidemic intelligence, vaccine preventable diseases and migrants and Cross-border emerging zoonoses which are expected 
to produce, among others, strategic documents and guidelines aimed at supporting the EpiSouth countries in setting standard operating procedures and in dealing with emerging health threats. Training modules on field/applied epidemiology intend to strengthen the capacities in the areas mentioned above.

A project steering committee identifies priorities, guides and advises the project coordinating structure. It is composed of the WP leaders plus observing representatives of EC Directorate-General for Health and Consumer Protection (DG Sanco), the European Centre for Disease Prevention and Control (ECDC), the Italian Ministry of Work, Health and Social Policies, the World Health Organization (WHO) and its Regional Office for Europe, Regional Office for the Eastern Mediterranean, and Lyon Office. All WHO entities provide input to the WPs' activities.

To enhance work in the WPs, steering teams (STs) were created. They consist of eight to nine experts, including the WP leader and representatives from European Union (EU) and non-EU countries that expressed interest in the respective WP. Finally, the network is constituted by one or two focal points (FP) formally appointed by each country. They interact with the relevant officers in their respective countries/organisations, with the other FPs of the network and with the project coordinator. FPs are expected to carry out, or promote and facilitate, all activities in the WPs requiring the countries' active involvement such as filling in questionnaires for assessment and evaluation, data and alerts sharing, contribution to documents and articles. According to their needs and interests countries can participate in the activities of one up to all WPs.

\section{Results and achievements}

\section{Participants}

Starting with nine EU countries, Italy, Spain, France, Bulgaria, Greece, Cyprus, Malta, Romania and Slovenia, EpiSouth has rapidly grown. As of December 2008, 26 countries (see figure) have appointed 63 country FPs, 29 from EU-countries and 34 from non-EU countries. Furthermore, seven experts represent collaborating institutions (see table) A directory of public health institutions participating in the network provides useful information on their respective mission, structure, role and activities in the field of infectious diseases prevention and control is available from the projects website [2]. The progress towards the network's building and development, was assessed through questionnaires distributed among the participants during the two annual meetings in Rome and Athens [3].

\section{Cross-border epidemic intelligence}

Consensus among EpiSouth partners was reached for the working definitions of epidemic intelligence: monitoring of health events occurring outside EpiSouth catchment area but with a possibility to affect EpiSouth countries and for cross-border issues: monitoring of health events occurring within the EpiSouth area for early warning and information sharing. Needs and expectations of participating countries towards this critical domain were assessed in a questionnaire that was elaborated by the ST members and distributed to all partners in November 2007. A report based on the outcomes was published on the public portal of the project's website [4]. It shows a common understanding of the perceived importance posed by emerging health threats throughout the EpiSouth catchment area. However, some differences were observed mainly related to historical structures of the surveillance systems (e.g. availability of adequate resources) and countries specificities (e.g. geographical location, history etc.). Although the number of returned questionnaires is not exhaustive, the analysis provided a solid base for elaborating EpiSouth international epidemic intelligence criteria both in terms of geographic coverage and type of health events potentially concerned.

Regarding regional cross-border issues, the assessment showed that most of the countries apply either national or international guidelines to report potential cross-border outbreaks. Concerning the implementation of a data platform exchange, half of the responding countries foresaw possible difficulties or restrictions to sharing information, particularly sharing of genuinely sensitive data.

Based on the needs and expectations identified in the survey, a pilot electronic EpiSouth Weekly Epi Bulletin (e-WEB) was elaborated and it is shared within the network since March 2008. Starting from summer 2008 a specific section of the bulletin is reporting events occurring in the EpiSouth area. In addition, direct exchange of information was initiated between the WP6 team and EpiSouth countries to add details or clarify information on health events occurring especially at cross-border level. The accessrestricted area on the website is going to be used as platform to allow confidential and rapid exchange of information and alerts from and restricted to the partners in the EpiSouth network. Thematic notes on specific diseases or events were produced on an ad hoc basis, one of them in collaboration with WHO [5].

\section{Vaccine-preventable diseases and migrants}

A provisional survey has been carried out among the seven WP steering team countries to collect the following set of information: type and size of migrant populations in the countries; immunisation programmes; assessment of vaccination coverage; availability of programmes ensuring high vaccination coverage of migrants; surveillance of vaccine-preventable diseases (VPD) in migrants. The results were internally shared in February 2008 and have guided the expanded multi-country on-line assessment among all 26 countries of EpiSouth network.

In February 2008 a workshop was held in Sofia, organised by ECDC and WHO-EURO in collaboration with EpiSouth and the EU surveillance network for vaccine-preventable diseases (EUVAC. NET), with the aim of improving the immunisation coverage in hard-to-reach target groups, particularly Roma communities in eastern Europe, by providing participating countries with technical guidance on using communication and information in this respect.

\section{F I G U R E}

Countries participating in the EpiSouth network as of December $2008(n=26)$.

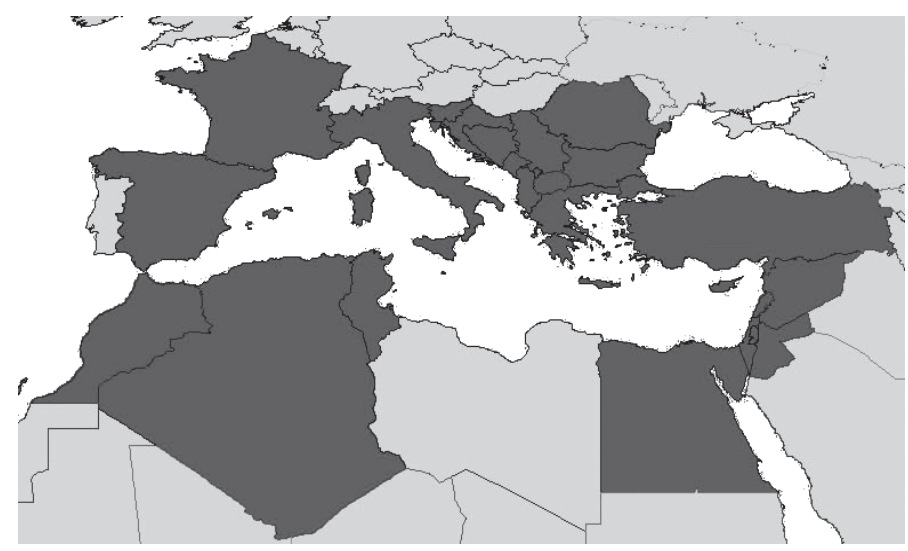


Special attention was paid to improving measles vaccination coverage in migrant population in these countries.

\section{Cross-border emerging zoonoses}

A dedicated questionnaire was developed for the selection of the priority zoonoses in the Episouth region.

The data, collected from 21 EpiSouth countries and analysed with specific criteria, have led to the formulation of the EpiSouth list of priority zoonotic diseases that includes: brucellosis, leishmaniasis, campylobacteriosis, rabies, West Nile virus infection [6]. The zoonoses selected cover at least two pathogens of public health importance for each participating country as well as a wide spectrum of diseases. These priority zoonoses are the basis for strengthening surveillance by describing the epidemiological situation in the area and for enhancing, among others, the collaboration and communication between human and veterinarian public health officials within each country and among countries of the EpiSouth network.

\section{Training}

The training modules programmes are based on results from a training needs assessment conducted in July 2007, through a questionnaire submitted to decision-makers/epidemiologists in all the countries of the network [7]. This assessment showed that participating institutions tend to be understaffed at central level and lack trained professionals. Furthermore, it revealed that in most of the responding countries, less than $25 \%$ of personnel in surveillance had received training in the past two years and that priority topics for training were quantitative risk assessment, modelling to understand dispersion of environmental risks and

\section{T A B L E}

Countries, Organisations and institutions participating in the EpiSouth network as of December 2008.

\begin{tabular}{|c|c|}
\hline Country or Organisation, location & Institution \\
\hline ALBANIA - Tirana & Institute of Public Health \\
\hline ALGERIA - Alger & National Institute of Public Health \\
\hline BOSNIA and HERZEGOVINA - Sarajevo, Banja Luka, Mostar & $\begin{array}{l}\text { Public Health Institute, Banja Luka, Republica Srpska } \\
\text { Ministry of Health of Federation of Bosnia and Herzegovina, Mostar } \\
\text { Ministry of Civil Affairs, Sarajevo }\end{array}$ \\
\hline BULGARIA - Sofia & National Center of Infectious and Parasitic Diseases - NCIPD \\
\hline CROATIA - Zagreb & Croatian National Institute of Public Health \\
\hline CYPRUS - Nicosia & Ministry of Health \\
\hline EGYPT - Cairo & Ministry of Health and Population \\
\hline FORMER YUGOSLAV REPUBLIC OF MACEDONIA - Skopje & Institute for Health Protection and Clinic of Infectious Diseases \\
\hline FRANCE - Saint Maurice Cedex & French Institute for Public Health Surveillance - InVS \\
\hline GREECE - Athens & Hellenic Center for Diseases Control and Prevention - HCDCP \\
\hline ISRAEL - Jerusalem & Israel Center for Disease Control Tel Hashomer and Ministry of Health Jerusalem \\
\hline ITALY - Rome, Padua & $\begin{array}{l}\text { Italian National Institute of Health - ISS, Rome } \\
\text { Padua Teaching Hospital, Padua }\end{array}$ \\
\hline JORDAN, Amman & Ministry of Health \\
\hline KOSOVO (UNSCR 1244) - Pristina & National Institute of Public Health \\
\hline LEBANON - Beirut & Ministry of Public Health \\
\hline MALTA - Msida & Ministry of Social Policy \\
\hline MONTENEGRO - Podgorica & Institute of Public Health \\
\hline MOROCCO - Rabat & Ministry of Health and National Institute of Hygiene \\
\hline PALESTINE - Ramallah & Ministry of Health \\
\hline ROMANIA - Bucharest & Institute of Public Health - IPH/ISPB \\
\hline SERBIA - Belgrade & Institute of Public Health of Serbia \\
\hline SLOVENIA - Ljubljana & Institute for Public Health of the Republic of Slovenia - NIPH/IVZ-RS \\
\hline SPAIN - Madrid & Health Institute Carlos III \\
\hline SYRIA - Damascus & Ministry of Health \\
\hline TUNISIA - Tunis & Ministry of Health \\
\hline TURKEY - Ankara & Ministry of Health \& Refik Saydam Hygiene Center \\
\hline European Commission - Luxembourg & Directorate-General for Health and Consumer Protection (DG Sanco) \\
\hline European Community agency - Stockholm & European Centre for Disease Prevention and Control (ECDC) \\
\hline ITALY - Rome & Ministry for Work, Health and Social Policies \\
\hline WHO - Lyon & World Health Organization Headquarters, Lyon office - WHO HQ/LYO \\
\hline WHO - Copenhagen & World Health Organization Regional office for Europe - WHO EURO \\
\hline WHO - Cairo & World Health Organization Regional office for Eastern Mediterranean - WHO EMRO \\
\hline
\end{tabular}


infectious diseases dynamics, epidemic intelligence and advanced data analysis.

So far, two training modules were conducted in September 2007 and June 2008, with the participation of 33 and 30 experts from 18 countries and 20 countries respectively, with $60 \%$ of participants from non-EU countries. Both training modules were anticipated by a workshop session were participants reported and shared experiences concerning the training topics.

\section{The EpiSouth website and data dissemination}

The EpiSouth website, launched in March 2007, contains a public portal devoted to the dissemination of information and results generated by EpiSouth and an access-restricted network working area to support the work of the project participants by enabling direct sharing and discussion of information [2]. Through the public website relevant guidelines, documents, and policies on topics of interest for the project as well as links to useful national and international organisations are made available. The official language of EpiSouth, and thus the website, is English. However, documents in French or Arabic, both languages spoken in more than one fourth of participating countries, are posted on the public web site without translation.

During the second year of activities (October 2007-September 2008), there were 9,079 unique visitors accounting for 14,895 visits on the public area of the website. Both the number of visitors and the number of visits have increased since the launch of the website (first year of activities: monthly average number of visitors and visits 173 and 399 respectively; second year of activities: 757 and 1,241). Furthermore, in 2008, the access-restricted network working area was accessed at least once by 64 (79\%) of the 81 authorised users. A total of 150 documents were uploaded, a monthly average of 12.5 documents compared with 9.0 documents in the first year of activities.

In September 2007 the first issue of the EpiSouth electronic bulletin summarising the latest information presented in the website, was released and sent to 500 recipients. Further issues were published in January, May, July and November 2008, the latest of which was sent to 592 recipients in 51 countries, an increase of almost $20 \%$ in recipients compared to the first issue [8].

\section{Conclusions}

EpiSouth has been successfully positioned in the international context through a wide range of communication activities and networking at meetings and conferences. It has attracted many different countries in the Mediterranean region and the Balkans (26 countries participate) as well as distinguished international partners. The network has contributed to building mutual trust and understanding which are an important basis for creating stability and collaboration in case of a public health emergency. Some of the challenges that lie ahead are related to project timelines and targets and to the sustainability of the project beyond 2009. So far however, there has been a growing capacity to network among the EpiSouth countries. The progressive acquaintance with the networking tools made available via the EpiSouth website and the enthusiastic collaboration in the context of the WP steering teams are indicators for an increasing sense of ownership among participants. In this respect it is necessary to consider that this project is peculiar. It covers a geographical area, the Mediterranean Basin and the Balkans, which as a whole is neither addressed by any EU network nor by WHO, as it encompasses both EU and non-EU countries and countries belonging to three different WHO regional offices. In this framework, efforts need to be performed to critically define all activities with the aim of enhancing work done by other institutions by filling gaps and avoiding overlapping or duplication of work. The EU, ECDC and WHO participation in the project steering committee is actually helping this process.

The EpiSouth network is in line with the EU neighbourhood policy and the Euro-Mediterranean partnership which identifies networks as tools for cooperation and integration with neighbouring countries. Well beyond the duration of the EpiSouth project, EpiSouth could effectively act as a bridge among countries that are epidemiologically linked but jurisdictionally separated.

\section{Aknowledgements}

We acknowledge the contribution to the project development of M. Ciotti (ECDC), P. Nabeth (WHO-HQ/LYO), N. Emirouglu and D. Mercer (WHO-EURO), J. Jabbour and J. Majour (WHO-EMRO) who have actively participated in the project's meetings and steering committee meetings as members of the network and as observers of the steering committee. We are also grateful to Flavia Riccardo for her contribution to the drafting of this article.

The project receives funds from the EU Public Health Programme (EC-DG Sanco) and the Italian Ministry of Work, Health and Social Policies (through the EpiMed Project). In addition, the financial support of EC EuropeAid and DG Enlargement (through the TAIEX facility) has made the participation of non-EU Countries to the 1st and 2nd EpiSouth Project Meetings possible.

\section{References}

1. EpiNorth [homepage on the internet]. Oslo: Folkehelseinstitut. Available from: www.epinorth.org

2. EpiSouth. WP4 - Institution partners data search. Rome: Istituto Superiore di Sanità. Available from: www.episouth.org/all_partners.html.

3. Gnesotto R, Putoto G, Montagna C, Dente MG, Fabiani M, Declich S. Monitoring Tool of Network Development: results from the questionnaire distributed during the First and the Second Project Meetings EpiSouth report 3/2008. Available from: www.episouth.org/outputs/wp3/2nd Monitoring_of_EpiSouth_Network_Development_ATHENS.pdf

4. Aït-Belghiti F, Barboza P and the WP6 steering team. Cross-Border Epidemic Intelligence evaluation: Results from the questionnaire on countries' needs and Expectations. EpiSouth report 5/2008. Available from: www.episouth.org/ outputs/wp6/WP6_Report-Assessment_survey-FINAL.pdf

5. EpiSouth, World Health Organization Regional Office for Europe. Epidemiology of Crimean-Congo haemorrhagic fever virus: Albania, Bulgaria, Greece, Islamic Republic of Iran, Kosovo, Russian Federation, Turkey. 1st October 2008. Available from: www.episouth.org/outputs/wp6/Episouth_CCHF_01_10_2008 final.pdf

6. Vorou R, Mellou K, Dougas G, Gkolfinopoulou K, Papamichail D, Papadimitriou T et al. Selection of zoonoses of priority in the Episouth countries: final report on the assessment conducted. EpiSouth report 4/2008. Available from: www. episouth.org/outputs/wp8/WP8Report_Public_area_FINALE_REV_9-4-08.pdf

7. Savalescu C, El Omeiri N, Martin de Pando1 C, Simon Soria F and the WP5 steering team. Report of Training Needs Assessment in Countries Participating in the EpiSouth Project. EpiSouth report 6/2008. Available from: www.episouth. org/outputs/wp5/WP5-survey_Report_v_Fin.pdf

8. EpiSouth. WP2 - EpiSouth Electronic Bulletin. Rome, Italy: EpiSouth; 2007/8. Available from: www.episouth.org/epi_ebulletin.htm?

This article was published on 5 February 2009.

Citation style for this article: Dente MG, Fabiani M, Gnesotto R, Putoto G, Montagna C Simon-Soria F, Martin de Pando C, Barboza P, Ait-Belghiti F, Kojouharova M, Vladimirova N, Vorou R, Mellou K, Thinus G, Declich S, for the EpiSouth Network. EpiSouth: a network for communicable disease control in the Mediterranean region and the Balkans. Euro Surveill. 2009;14(5):pii=19113. Available online: http://www.eurosurveillance.org/ ViewArticle.aspx?ArticleId=19113 